\title{
Pseudosarcomatous myofibroblastic proliferations of the genitourinary tract are genetically different from nodular fasciitis and lack USP6, ROS1 and ETV6 gene rearrangements
}

\author{
Judith A S Jebastin, ${ }^{1}$ Steven C Smith, ${ }^{2}$ Kyle D Perry, ${ }^{1}$ Nilesh S Gupta, ${ }^{1}$ Shaheen Alanee, ${ }^{3}$ \\ Shannon Carskadon, ${ }^{3}$ Dhananjay A Chitale, ${ }^{1,4}$ Nallasivam Palanisamy $^{3,5}$ \& \\ Sean R Williamson ${ }^{1,4}$ iD \\ ${ }^{1}$ Department of Pathology and Laboratory Medicine and Henry Ford Cancer Institute, Henry Ford Health System, \\ Detroit, MI, ${ }^{2}$ Department of Pathology, Virginia Commonwealth University, Richmond, VA, ${ }^{3}$ Vattikutti Urology \\ Institute, Henry Ford Health System, ${ }^{4}$ Department of Pathology, Wayne State University School of Medicine, Detroit, \\ and ${ }^{5}$ Michigan Center for Translational Pathology, University of Michigan, Ann Arbor, MI, USA
}

Date of submission 28 February 2018

Accepted for publication 29 March 2018

Published online Article Accepted 4 April 2018

Jebastin J A S, Smith S C, Perry K D, Gupta N S, Alanee S, Carskadon S, Chitale D A, Palanisamy N \&

Williamson S R

(2018) Histopathology 73, 321-326. https://doi.org/10.1111/his.13526

Pseudosarcomatous myofibroblastic proliferations of the genitourinary tract are genetically different from nodular fasciitis and lack USP6, ROS1 and ETV6 gene rearrangements

Aims: Pseudosarcomatous myofibroblastic proliferations of the genitourinary tract have a debatable relationship with inflammatory myofibroblastic tumour (generally lacking $A L K$ rearrangement); however, they share several overlapping features with nodular fasciitis of soft tissue. As rearrangement of the USP6 gene has been recently recognised as a recurrent alteration in soft tissue nodular fasciitis, and several other alternative gene fusions have been recently recognised in inflammatory myofibroblastic tumour, the aim of this study was to investigate whether USP6, ROS1 or ETV6 rearrangements were present in these lesions (12 cases).

Methods and results: Fluorescence in-situ hybridisation analysis was performed by the use of bacterial artificial chromosome-derived break-apart probes against USP6, ROS1, and ETV6. Two cases with adequate genetic material from recent paraffin tissue blocks were also tested by use of a solid tumour gene fusion detection assay via next-generation sequencing, targeting $>50$ known genes involved in recurrent fusions. None of the genitourinary pseudosarcomatous myofibroblastic proliferations was found to harbour USP6 (0/12), ROS1 (0/8) or ETV6 (0/7) rearrangements, and no gene fusions were detected in two cases studied by sequencing.

Conclusions: Despite overlap in histological and immunohistochemical features between pseudosarcomatous myofibroblastic proliferation and nodular fasciitis, these tumours lack the recently recognised USP6 rearrangements that occur in nodular fasciitis, as well as alternative fusions found in $A L K$-negative inflammatory myofibroblastic tumours. At present, this diagnosis remains based primarily on clinical, histological and immunohistochemical features.

Keywords: inflammatory myofibroblastic tumour, pseudosarcomatous myofibroblastic proliferation, soft tissue tumours, urinary bladder, USP6 


\section{Introduction}

Pseudosarcomatous myofibroblastic tumours of the genitourinary tract, ${ }^{1}$ whether procedure-related or spontaneous, can pose diagnostic challenges for pathologists, owing to one or more worrying features, such as high mitotic rate, an infiltrative growth pattern, recurrence, and overlapping morphological and immunophenotypic features with other spindle cell tumours, such as low-grade leiomyosarcoma and sarcomatoid carcinoma. These tumours are also known by a variety of names, including fibromyxoid pseudotumour and postoperative spindle cell nodule, and it remains controversial whether this and inflammatory myofibroblastic tumour ${ }^{2,3}$ are the same entity or different entities. ${ }^{4-7}$ Regardless of the terminology used, both pseudosarcomatous myofibroblastic proliferations and inflammatory myofibroblastic tumours of the urinary tract generally show non-aggressive behaviour without metastasis, although some cases have persisted or recurred, requiring multiple surgical procedures. Therefore, recognition is important, as the differential diagnosis for spindle cell tumours of the urinary tract should almost always include consideration of sarcomatoid carcinoma and sarcoma, including leiomyosarcoma.

As rearrangement of the ubiquitin-specific protease 6 (USP6) gene has been recently recognised as a recurrent alteration in soft tissue nodular fasciitis, ${ }^{8}$ and as pseudosarcomatous myofibroblastic proliferation of the genitourinary tract has a number of similarities with nodular fasciitis, including labelling for myofibroblastic marker smooth muscle actin, we aimed to evaluate USP6 rearrangement in pseudosarcomatous myofibroblastic tumours of the urinary tract, in order to determine whether this may be a confirmatory diagnostic test for this uncommon diagnosis, making it the genitourinary tract equivalent of nodular fasciitis. Additionally, we also studied ROS1 and ETV6 for rearrangement, as these have been recently implicated in a subset of $A L K$ rearrangement-negative inflammatory myofibroblastic tumours. ${ }^{9,10}$ Two representative cases with the most optimal genetic material were also tested with a gene fusion detection panel targeting $>50$ genes involved in recurrent gene fusions, by the use of next-generation sequencing.

\section{Materials and methods}

After obtaining institutional review board approval from the Henry Ford Health System, we retrieved 17 cases of pseudosarcomatous myofibroblastic proliferation of the genitourinary tract from our pathology databases from 2002 to 2017, none of which was included in a prior study of inflammatory myofibroblastic tumours. ${ }^{11}$ of the 17 cases, four were reclassified upon histological review. One occurred in the inguinal canal; as this is a non-visceral location, this case was excluded. We studied the remaining 12 cases of pseudosarcomatous myofibroblastic proliferation, 10 of which occurred in the urinary bladder and two in the renal pelvis. Histopathology and immunohistochemical staining results were reviewed by two of the authors (J.A.S.J. and S.R.W.). We included pseudosarcomatous myofibroblastic proliferations with the following histological and immunohistochemical features. Lesions were required to be composed of spindle-shaped cells arranged in compact fascicles or with a loose tissue culture-like appearance. The spindle-shaped cells were required to be bipolar with eosinophilic cytoplasm, and uniform ovoid or spindle-shaped nuclei with smooth nuclear contours. Stromal changes included myxoid change, areas of collagenisation, haemorrhage, and scattered inflammatory cells. As is the diagnosis of nodular fasciitis, a specific cut-off of mitotic activity was not employed; however, an absence of overt cytological atypia, atypical mitosis, and necrosis was required. The expected immunohistochemical phenotype included positivity for smooth muscle actin or muscle-specific actin, and negativity for p63, GATA3, and high molecular weight cytokeratin. Variable staining for desmin and broad-spectrum cytokeratin was allowed, in light of previous reports showing these findings. ${ }^{12,13}$ If not already performed at the time of original diagnosis, ALK immunohistochemical staining was performed with anti-ALK antibody (monoclonal mouse anti-human CD246, ALK protein, clone ALK-1; Dako, Carpinteria, CA, USA). As ALK rearrangement is rare in cases with negative immunohistochemical staining, ${ }^{1,11,14}$ we did not perform ALK fluorescence in-situ hybridisation (FISH).

\section{F I S H}

FISH analyses for USP6, ROS1 and ETV6 were performed with bacterial artificial chromosome-derived break-apart probes. FISH for USP6 was validated to be positive in four well-characterised cases of nodular fasciitis (Figure 1A,B) and one aneurysmal bone cyst, and negative in eight histological mimics of nodular fasciitis. ROS1 and ETV6 FISH was performed on all 12 cases, but lacked adequate hybridisation signals in five cases each for ROS1 and ETV6, even after repeated hybridisation. One case that was non- 
informative for ROS1 by FISH was informative by the next-generation sequencing assay, bringing the totals of ROS1 and ETV6 cases analysed to eight of 12 and seven of 12 , respectively.

\section{NEXT - GENER A TI ON SEQUENCING}

A multiplex RNA fusion panel (Archer FusionPlex Solid Tumour Kit, ArcherDX, Inc, Boulder, CO, USA) was applied to RNA extracted from representative formalin-fixed paraffin-embedded tissue tumour blocks of two of the most recent cases regarded as having the most adequate genetic material for assessment $(<2$ years since tissue collection). Briefly, this assay is a targeted sequencing assay that uses anchored multiplex polymerase chain reactions to prepare targetenriched cDNA libraries from RNA to detect fusions and other mutations in $>50$ genes linked to carcinomas and other solid tumours, known sarcoma and haematological malignancy-associated fusions and prominent BRAF and PDGFRA mutations by the use of next-generation sequencing. This technology allows the detection of not only known recurrent fusions, but also previously unidentified fusions, so long as one of the fusion partners is included in the panel. Anchored multiplex polymerase chain reaction creates target-enriched libraries by using a combination of unidirectional gene-specific primers and universal adapters to enrich for both known and unknown mutations. Adapters that contain both molecular barcode adapters and sample indices permit quantitative multiplex data analysis, read deduplication, and accurate mutation calling. Molecular barcode adapters aid in error correction, sample identification, deduplication, and duplicate read binning for confident mutation detection. Genes targeted in this assay include: AKT3, ALK, ARHGAP26, AXL, BRAF (fusion and V600E mutation), BRD3, BRD4, EGFR (fusion and mutation), ERG, ESR1, ETV1, ETV4, ETV5, ETV6, EWSR1, FGFR1, FGFR2, FGFR3, FGR, INSR, MAML2, MAST1, MAST2, MET (fusion and mutation), MSMB, MUSK, MYB, NOTCH1, NOTCH2, NRG1, NTRK1, NTRK2, NTRK3, NUMBL, NUTM1, PDGFRA (fusion and mutation), PDGFRB, PIK3CA, PKN1, PPARG, PRKCA, PRKCB, RAF1, RELA, RET, ROS1, RSPO2, RSPO3, TERT, TFE3, TFE3, TFEB, THADA, and TMPRSS2.

\section{Results}

The age distribution of the 12 patients studied ranged from 26 to 87 years (median 62.5 years); there were five men and seven women. Three of the 12 patients had recurrent or persistent tumour in more than one transurethral resection. One had multiple recurrences, which were ultimately managed with

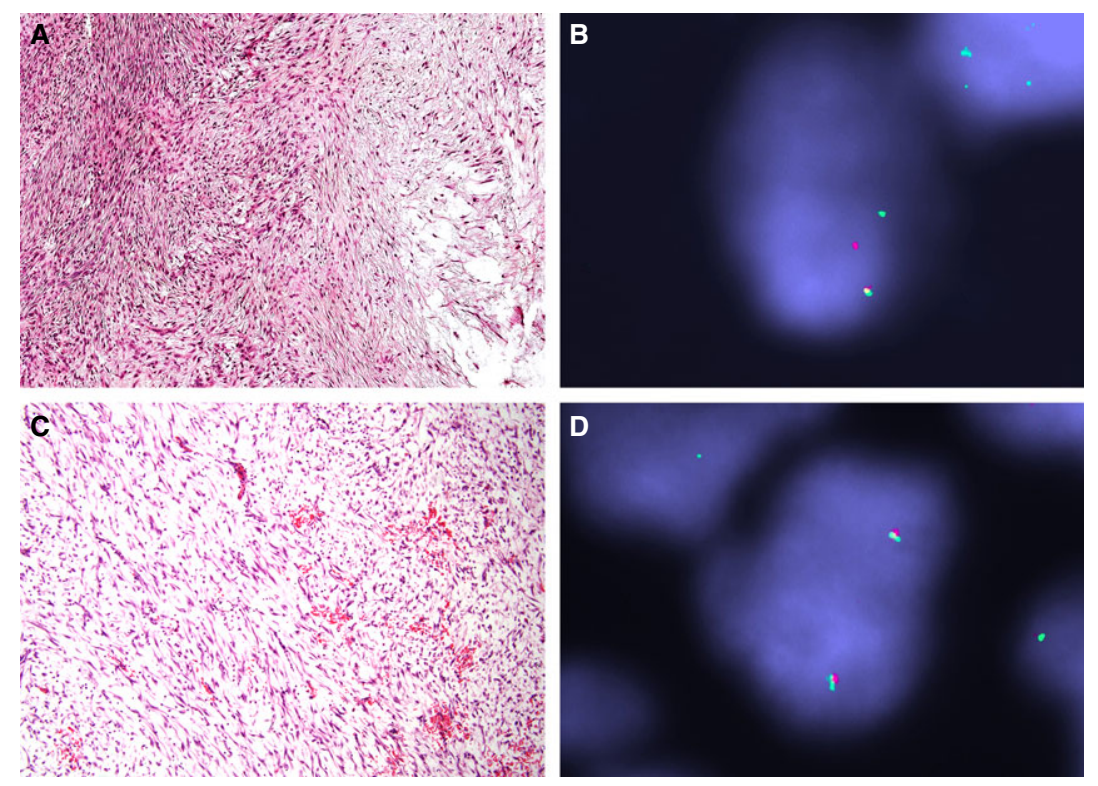

Figure 1. A, The histological appearance of a case of USP6-rearranged nodular fasciitis demonstrates fusiform cells with areas of myxoid oedematous stroma. B, Break-apart fluorescence in-situ hybridisation (FISH) shows rearrangement of USP6, with separation of one pair of green and red signals. C, Pseudosarcomatous myofibroblastic proliferation of the urinary bladder has a very similar morphological appearance. D, However, break-apart FISH is negative for USP6 rearrangement. 
cystectomy. Three of 10 patients had a known history of previous instrumentation in the bladder, and one had undergone prior uterine surgery. The histological findings were similar in all cases, irrespective of age, site, and recurrence status, as described in the criteria and Discussion. ALK immunohistochemical staining was negative in all cases. FISH analysis (Figure 1C,D) for USP6 gave negative results in all 12 cases $(0 / 12)$, including 10 large tumours [making up multiple tissue cassettes of lesional tissue, (Figure 2)] and two small lesions (entirely examined in one or two tissue cassettes). FISH analysis for ROS1 and ETV6 gave negative results in seven of the 12 cases $(0 / 7)$. The remaining cases lacked hybridisation signals even after repeated hybridisation. The next-generation sequencing revealed no identifiable gene fusions from the studied panel of $>50$ genes in two cases with adequate genetic material for evaluation, which included ROS1 in one of the cases that failed FISH (total 0/8 for ROS1).

\section{Discussion}

Pseudosarcomatous myofibroblastic proliferation is a descriptive term that encompasses the mucosal lesions postoperative spindle cell nodule, visceral fasciitis, pseudosarcomatous fibromyxoid tumour, pseudomalignant spindle cell proliferation, and inflammatory pseudotumour. ${ }^{1}$ In general, the term postoperative spindle cell nodule is principally used in the setting of previous injury or biopsy, in contrast to other terms used when the mass occurs idiopathically. These spindle-shaped cells show ultrastructural features of myofibroblasts intermediate between a fibroblast and smooth muscle cell. ${ }^{15,16}$ Although this has been reported in multiple mucosal sites, the urinary tract is among the most common locations. In addition to these several names, it also remains a subject of debate whether this entity is part of the spectrum of inflammatory myofibroblastic tumour. ${ }^{2-4}$ Both of these entities have very similar morphology, with, perhaps, the exception of a lesser inflammatory component in pseudosarcomatous myofibroblastic proliferation, and both have a myofibroblastic immunohistochemical phenotype. Although inflammatory myofibroblastic tumour of soft tissue is classified as a neoplasm of intermediate malignancy, both of these entities in the urinary tract are characteristically non-aggressive (without metastasis, although persistence or recurrence, requiring multiple surgeries, can occur). In general, our practice is to reserve the term inflammatory myofibroblastic tumour (in the genitourinary tract) predominantly for tumours
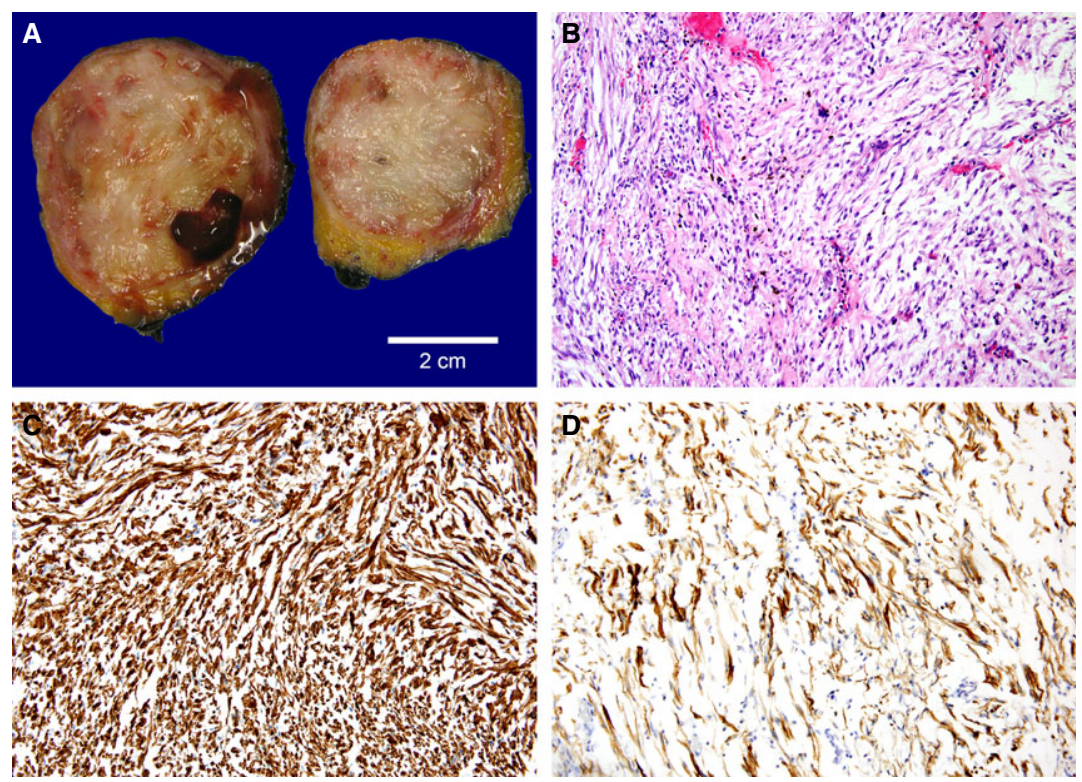

Figure 2. A, Gross appearance of a partial cystectomy specimen from a case of pseudosarcomatous myofibroblastic proliferation of the urinary bladder shows a solid, white mass in the wall of the urinary bladder. B, The same tumour shows nodular fasciitis-like histology. C, Immunohistochemical staining for smooth muscle actin demonstrates diffuse, strong staining, consistent with a myofibroblastic pattern. D, Keratin (broad-spectrum cocktail) immunohistochemical staining demonstrates substantial labelling, in contrast to classic soft tissue nodular fasciitis. 
with ALK immunohistochemical positivity or ALK rearrangement; however, even this is a subject of debate, as some studies have found that cases with positive ALK immunohistochemical staining do not necessarily always harbour $A L K$ rearrangement, making this distinction imprecise. ${ }^{1,4,5,11}$

Histologically, pseudosarcomatous myofibroblastic proliferations are composed of proliferations of spindle-shaped cells with loose myxoid stroma or tight fascicles. They have variable degrees of mitotic activity and inflammatory cell infiltrate. However, marked pleomorphism, atypical mitosis and necrosis are absent. Immunohistochemically, these typically stain for muscle-specific actin, smooth muscle actin (Figure 2C), sometimes desmin, and often cytokeratin (Figure 2D). Overall, these could be argued to resemble soft tissue nodular fasciitis in both histology and reactivity for smooth muscle actin; however, labelling for keratin is typically a point of distinction that is frequently present in bladder myofibroblastic tumours but lacking in nodular fasciitis. ${ }^{17,18}$ Despite the fact that nodular fasciitis has long been considered to be an often self-limiting exuberant reactive lesion, clonal rearrangement of the USP6 locus at $17 \mathrm{p} 13$ resulting in MYH9-USP6 fusion has been recently identified, suggesting that, rather than being a purely reactive process, this may in fact represent a transient form of neoplasia. It is of note that nodular fasciitis also shares with pseudosarcomatous myofibroblastic proliferation of the urinary tract an incomplete association with preceding trauma. Therefore, in soft tissue tumour pathology, evaluation for USP6 rearrangement may be a potentially helpful technique to resolve challenging diagnostic cases. ${ }^{19}$ Interestingly, rearrangement of the USP6 locus is also shared by aneurysmal bone cyst, ${ }^{20}$ which is histologically quite different, although perhaps less so if one considers the bland spindle-shaped cell component of aneurysmal bone cyst to be the true neoplastic cell population. Despite the histological, immunophenotypic, and ultrastructural ${ }^{16,21,22}$ similarities between pseudosarcomatous myofibroblastic proliferation of the genitourinary tract and nodular fasciitis, we found that these lesions lacked USP6 rearrangement (Figure 1D), which refutes our hypothesis that this may be its visceral equivalent.

The staining for cytokeratin that is often present in pseudosarcomatous myofibroblastic proliferation may also raise consideration of sarcomatoid urothelial carcinoma; however, features than can be helpful in distinguishing these entities include reactivity for p63 or GATA3 in sarcomatoid carcinoma, and a usual lack of reactivity for desmin and a limited extent of labelling for smooth muscle actin in sarcomatoid carcinoma. ${ }^{23}$ Although cytokeratin staining is often present in pseudosarcomatous myofibroblastic proliferation, staining for high molecular weight cytokeratin is characteristically absent, contrasting with urothelial carcinoma. ${ }^{24}$

A limitation of this study is that the FISH and sequencing testing employed were targeted only at known gene fusions, and was therefore not designed to entirely identify novel gene fusions. Further studies, such as with RNA sequencing or other techniques, may determine whether these pseudosarcomatous myofibroblastic proliferations of the urinary tract represent a benign neoplasm, like nodular fasciitis, but with a so far unknown gene fusion, or whether they represent an exuberant reactive process, as nodular fasciitis was originally postulated to be. On the basis of our current data, this diagnosis remains principally based on morphological and immunohistochemical features, without a known confirmatory molecular feature. If these rearrangements ever occur in pseudosarcomatous myofibroblastic proliferations of the urinary tract, their rate is probably sufficiently low that testing is not a robust diagnostic tool.

\section{Acknowledgements}

The authors would like to thank Dr Anthony Gill, University of Sydney and Royal North Shore Hospital, Sydney, Australia, for discussions about the project and the suggestion to also evaluate ROS1 and ETV6. No funding was received for this study. This work was presented, in part, at the United States and Canadian Academy of Pathology (USCAP) Annual Meeting, 2017.

\section{Conflicts of interest}

The authors state that they have no conflicts of interest.

\section{Author contributions}

Drafting of the manuscript: J. A. S. Jebastin. Critical revision and final approval of the manuscript: all authors. Data collection, analysis, and interpretation: J. A. S. Jebastin, S. Carskadon, D. A. Chitale, N. Palanisamy, and S. R. Williamson. Conception/design: S. R. Williamson.

\section{References}

1. Harik LR, Merino C, Coindre JM, Amin MB, Pedeutour F, Weiss SW. Pseudosarcomatous myofibroblastic proliferations of the 
bladder: a clinicopathologic study of 42 cases. Am. J. Surg. Pathol. 2006; 30; 787-794.

2. Montgomery EA, Shuster DD, Burkart AL et al. Inflammatory myofibroblastic tumors of the urinary tract: a clinicopathologic study of 46 cases, including a malignant example inflammatory fibrosarcoma and a subset associated with high-grade urothelial carcinoma. Am. J. Surg. Pathol. 2006; 30; 15021512.

3. Tsuzuki T, Magi-Galluzzi C, Epstein JI. ALK-1 expression in inflammatory myofibroblastic tumor of the urinary bladder. Am. J. Surg. Pathol. 2004; 28; 1609-1614.

4. Cheng L, Foster SR, MacLennan GT, Lopez-Beltran A, Zhang S, Montironi R. Inflammatory myofibroblastic tumors of the genitourinary tract-single entity or continuum? J. Urol. 2008; 180; $1235-1240$.

5. Hirsch MS, Dal Cin P, Fletcher CD. ALK expression in pseudosarcomatous myofibroblastic proliferations of the genitourinary tract. Histopathology 2006; 48; 569-578.

6. Williamson SR, Lopez-Beltran A, MacLennan GT, Montironi R, Cheng L. Unique clinicopathologic and molecular characteristics of urinary bladder tumors in children and young adults. Urol. Oncol. 2013; 31; 414-426.

7. Lott S, Lopez-Beltran A, Maclennan GT, Montironi R, Cheng L. Soft tissue tumors of the urinary bladder, Part I: myofibroblastic proliferations, benign neoplasms, and tumors of uncertain malignant potential. Hum. Pathol. 2007; 38; 807-823.

8. Erickson-Johnson MR, Chou MM, Evers BR et al. Nodular fasciitis: a novel model of transient neoplasia induced by MYH9USP6 gene fusion. Lab. Invest. 2011; 91; 1427-1433.

9. Yamamoto H, Yoshida A, Taguchi $\mathrm{K}$ et al. ALK, ROS1 and NTRK3 gene rearrangements in inflammatory myofibroblastic tumours. Histopathology 2016; 69; 72-83.

10. Cook JR, Dehner LP, Collins MH et al. Anaplastic lymphoma kinase (ALK) expression in the inflammatory myofibroblastic tumor: a comparative immunohistochemical study. Am. J. Surg. Pathol. 2001; 25; 1364-1371.

11. Choi E, Williamson SR, Montironi R et al. Inflammatory myofibroblastic tumour of the urinary bladder: the role of immunoglobulin G4 and the comparison of two immunohistochemical antibodies and fluorescence in-situ hybridization for the detection of anaplastic lymphoma kinase alterations. Histopathology 2015; 67; 20-38.

12. Alquati S, Gira FA, Bartoli V, Contini S, Corradi D. Low-grade myofibroblastic proliferations of the urinary bladder. Arch. Pathol. Lab. Med. 2013; 137; 1117-1128.
13. Hojo H, Newton WA Jr, Hamoudi AB et al. Pseudosarcomatous myofibroblastic tumor of the urinary bladder in children: a study of 11 cases with review of the literature. An Intergroup Rhabdomyosarcoma Study. Am. J. Surg. Pathol. 1995; 19; 1224-1236.

14. Cabillic F, Gros A, Dugay F et al. Parallel FISH and immunohistochemical studies of ALK status in 3244 non-small-cell lung cancers reveal major discordances. J. Thorac. Oncol. 2014; 9; 295-306.

15. Ro JY, el-Naggar AK, Amin MB, Sahin AA, Ordonez NG, Ayala AG. Pseudosarcomatous fibromyxoid tumor of the urinary bladder and prostate: immunohistochemical, ultrastructural, and DNA flow cytometric analyses of nine cases. Hum. Pathol. 1993; 24; 1203-1210.

16. Iczkowski KA, Shanks JH, Gadaleanu V et al. Inflammatory pseudotumor and sarcoma of urinary bladder: differential diagnosis and outcome in thirty-eight spindle cell neoplasms. Mod. Pathol. 2001; 14; 1043-1051.

17. Lazar A, Evans HL, Oliveira AM. Nodular fasciitis. In Fletcher CDM, Bridge JA, Hogendoorn PC, Mertens F eds. World Health Organization classification of tumours of soft tissue and bone. Lyon: IARC Press, 2013; 46-47.

18. Koirala TR, Hayashi K, Ohara N et al. Inflammatory pseudotumor of the urinary bladder with an aberrant expression of cytokeratin. Pathol. Int. 1994; 44; 73-79.

19. Shin C, Low I, Ng D, Oei P, Miles C, Symmans P. USP6 gene rearrangement in nodular fasciitis and histological mimics. Histopathology 2016; 69; 784-791.

20. Oliveira AM, Perez-Atayde AR, Dal Cin P et al. Aneurysmal bone cyst variant translocations upregulate USP6 transcription by promoter swapping with the ZNF9, COL1A1, TRAP150, and OMD genes. Oncogene 2005; 24; 3419-3426.

21. Albores-Saavedra J, Manivel JC, Essenfeld H et al. Pseudosarcomatous myofibroblastic proliferations in the urinary bladder of children. Cancer 1990; 66; 1234-1241.

22. Wirman JA. Nodular fasciitis, a lesion of myofibroblasts: an ultrastructural study. Cancer 1976; 38; 2378-2389.

23. Sanfrancesco J, McKenney JK, Leivo MZ, Gupta S, Elson P, Hansel DE. Sarcomatoid urothelial carcinoma of the bladder: analysis of 28 cases with emphasis on clinicopathologic features and markers of epithelial-to-mesenchymal transition. Arch. Pathol. Lab. Med. 2016; 140; 543-551.

24. Westfall DE, Folpe AL, Paner GP et al. Utility of a comprehensive immunohistochemical panel in the differential diagnosis of spindle cell lesions of the urinary bladder. Am. J. Surg. Pathol. 2009; 33; 99-105. 